\title{
Exploring Citizen Psych-Science and the Motivations of Errordiary Volunteers
}

CHARLENE JENNETT, University College London, UK

DOMINIC FURNISS, University College London, UK

IOANNA IACOVIDES, University College London, UK

SARAH WISEMAN, University College London, UK

SANDY J. J. GOULD, University College London, UK

ANNA L. COX, University College London, UK

\begin{abstract}
Although virtual citizen science projects have the potential to engage large networks of people in science research, seeding and maintaining such networks can be difficult. A feature of successful projects is that they have well-motivated volunteers. What makes volunteers motivated rather than apathetic? In this paper we focus on projects that contribute to psychology research, which we term 'citizen psych-science'. This differs from typical citizen science because volunteers are asked to contribute themselves as data. We describe research studies that we conducted with Errordiary - a citizen psych-science project where volunteers tweet about their everyday experiences of human error. These studies were: (1) an interview study, to explore the motivations of eight Errordiary volunteers; and (2) three focus groups, to explore the potential of attracting new communities to Errordiary. We found that the personal nature of the data can influence participation in positive and negative ways. We suggest several factors that scientists need to consider when encouraging citizen psych-science volunteers to contribute their personal experiences towards research.
\end{abstract}




\section{INTRODUCTION}

Virtual citizen science is a type of crowdsourcing in which members of the public collaborate online with professional researchers to conduct scientific research. Sometimes this involves collecting environmental data outdoors, perhaps taking photos of animals or collecting noise readings. Other times volunteers might be analyzing data that requires human cognition because it is too complex to be processed by a machine. Identifying species, classifying galaxies and transcribing handwriting are activities that fall into this group.

The majority of virtual citizen science projects contribute to research in the biological and physical sciences. Topics include astronomy (Galaxy Zoo), biodiversity (eBird, iSpot, Project Noah), air pollution (WideNoise) and climate change (Old Weather). However we are beginning to see growing numbers of projects in other research domains, including neuroscience (Eyewire, Foldit), English literature (Transcribe Bentham), history (Ancient Lives) and psychology (Mappiness, Errordiary, Lab in the Wild).

In this paper we focus specifically on virtual citizen science projects that contribute to psychology research, which we term 'citizen psych-science'. Citizen psych-science differs from typical citizen science projects because volunteers are asked to contribute data that arise from their personal characteristics, rather than those of the physical environment. There is a shift from a third person narrative where we learn about the world in which we live, to a first person narrative where there is a greater potential to learn about ourselves and how we fit into the world. This is an important difference because, as our results will reveal, the personal nature of the data collected in citizen psych-science research can influence participation in positive and negative ways.

First we will present a brief overview of existing research investigating motivations in citizen science. Then we will describe Errordiary, a citizen psych-science project where volunteers tweet about their everyday errors. We will describe and discuss the findings of two research studies that we conducted with Errordiary. Finally we will conclude by suggesting factors that scientists need to consider when encouraging citizen psych-science volunteers to contribute their personal experiences towards research.

\section{BACKGROUND}

\subsection{Motivations in Virtual Citizen Science}

In virtual citizen science projects, scientists can potentially recruit help from a large network of people (Wiggins \& Crowston, 2011). However, attracting and maintaining such a network can be difficult. To tackle this problem, scientists have started conducting research to understand the 
motivations of volunteers. In Table 1 we provide a summary of 10 recent studies that have explored volunteers' motivations for participating in virtual citizen science.

Table 1. Previous research investigating motivations in citizen science.

\begin{tabular}{|c|c|c|c|}
\hline Authors & Method & Research Topics & Motivation Categories \\
\hline Raddick et al., 2010 & $\begin{array}{l}\text { Forum survey with } \\
\text { Galaxy Zoo } \\
\text { volunteers ( } 826 \text { forum } \\
\text { responses) and } \\
\text { interviews with } 22 \\
\text { volunteers }\end{array}$ & Astronomy & $\begin{array}{l}\text { Contribute, learning, } \\
\text { discover, community, } \\
\text { teaching, beauty, fun, } \\
\text { vastness, helping, zoo, } \\
\text { astronomy, science. }\end{array}$ \\
\hline Nov et al., 2010 & $\begin{array}{l}\text { Online survey with } \\
\text { SETI@home } \\
\text { volunteers and the } \\
\text { Citizen Science } \\
\text { Weather observation } \\
\text { program (number of } \\
\text { participants not } \\
\text { reported) }\end{array}$ & $\begin{array}{l}\text { Extraterrestial } \\
\text { intelligence, Climate }\end{array}$ & $\begin{array}{l}\text { SETI@ home: } \\
\text { enhancement, } \\
\text { affiliation to a team. } \\
\text { Citizen Science } \\
\text { Weather Observation } \\
\text { program: learning } \\
\text { new information, } \\
\text { values, norms and } \\
\text { intrinsic motivations. }\end{array}$ \\
\hline Nov et al., 2011 & $\begin{array}{l}\text { Online survey with } \\
139 \text { Stardust@home } \\
\text { volunteers }\end{array}$ & $\begin{array}{l}\text { Astronomy, } \\
\text { Extraterrestial } \\
\text { intelligence }\end{array}$ & $\begin{array}{l}\text { Collective, intrinsic, } \\
\text { identification, norm- } \\
\text { oriented. }\end{array}$ \\
\hline $\begin{array}{l}\text { Causer \& Wallace } \\
2012\end{array}$ & $\begin{array}{l}\text { Online survey with } \\
101 \text { Transcribe } \\
\text { Bentham volunteers }\end{array}$ & History, Literature & $\begin{array}{l}\text { Interest in } \\
\text { history/philosophy, } \\
\text { being part of } \\
\text { something } \\
\text { collaborative, interest } \\
\text { in the technology } \\
\text { and/or crowdsourcing, } \\
\text { interest in Bentham, } \\
\text { addition of new } \\
\text { material, competition, } \\
\text { and recognition. }\end{array}$ \\
\hline Rotman et al., 2012 & $\begin{array}{l}\text { Ecological citizen } \\
\text { science projects, } \\
\text { online survey with } \\
142 \text { volunteers and } \\
\text { scientists, interviews } \\
\text { with } 11 \text { volunteers }\end{array}$ & Nature & $\begin{array}{l}\text { Egoism, recognition } \\
\text { and attribution, } \\
\text { feedback, community } \\
\text { involvement, } \\
\text { advocacy. }\end{array}$ \\
\hline Crowston \& & Online survey with & Nature & Fun, interest in nature \\
\hline
\end{tabular}




\begin{tabular}{|c|c|c|c|}
\hline Prestopnik, 2013 & $\begin{array}{l}199 \text { Happy Match } \\
\text { players }\end{array}$ & & $\begin{array}{l}\text { activities, learning } \\
\text { about moths. }\end{array}$ \\
\hline Iacovides et al, 2013 & $\begin{array}{l}\text { Interviews with } 4 \\
\text { Foldit volunteers and } \\
4 \text { Eyewire volunteers }\end{array}$ & $\begin{array}{l}\text { Biology, } \\
\text { Neuroscience }\end{array}$ & $\begin{array}{l}\text { Interest in science, } \\
\text { pro-citizen science, } \\
\text { recognition, gaming } \\
\text { elements, and team- } \\
\text { play. }\end{array}$ \\
\hline Raddick et al., 2013 & $\begin{array}{l}\text { Online survey with } \\
\text { 10,992 Galaxy Zoo } \\
\text { volunteers }\end{array}$ & Astronomy & $\begin{array}{l}\text { Contribute, learning, } \\
\text { discover, community, } \\
\text { teaching, beauty, fun, } \\
\text { vastness, helping, zoo, } \\
\text { astronomy, science, } \\
\text { other. }\end{array}$ \\
\hline Reed et al., 2013 & $\begin{array}{l}\text { Online survey with } \\
199 \text { Zooniverse } \\
\text { volunteers }\end{array}$ & $\begin{array}{l}\text { Astronomy, History, } \\
\text { Biology, Nature, } \\
\text { Climate }\end{array}$ & $\begin{array}{l}\text { Social engagement, } \\
\text { interaction with } \\
\text { website, helping. }\end{array}$ \\
\hline Eveleigh et al., 2014 & $\begin{array}{l}\text { Online survey with } \\
299 \text { Old Weather } \\
\text { volunteers, qualitative } \\
\text { follow-up with } 17 \\
\text { volunteers }\end{array}$ & Climate, History & $\begin{array}{l}\text { Intrinsic motives (e.g. } \\
\text { subject interest, task } \\
\text { competence, } \\
\text { enjoyment), extrinsic } \\
\text { motives (e.g. } \\
\text { community, status). }\end{array}$ \\
\hline
\end{tabular}

It is evident that people are motivated to participate in virtual citizen science projects for many different reasons. These motivations include:

- Interest in the research topic (Raddick et al., 2010; Causer \& Wallace, 2012; Crowston \& Prestopnik, 2013; Reed et al,. 2013; Iacovides et al., 2013; Eveleigh et al., 2014)

- Learning new information (Raddick et al., 2010; Nov et al,. 2010; Crowston \& Prestopnik, 2013; Reed et al,. 2013)

- Contributing to original research (Raddick et al., 2013; Nov et al,. 2010; Reed et al, 2013,)

- Enjoying the research task (Raddick et al., 2010; Nov et al., 2010; Nov et al., 2011, Crowston \& Prestopnik, 2013; Reed et al, 2013; Eveleigh et al., 2014).

- Sharing the same goals and values as the project (Nov et al., 2010, Nov et al,. 2011)

- Helping others and feeling part of a team (Raddick et al, 2010; Nov et al,. 2010; Rotman et al., 2012; Nov et al., 2013; Iacovides et al., 2013; Reed et al, 2013).

- Receiving recognition and feedback (Rotman et al., 2012; Causer \& Wallace, 2012; Iacovides et al., 2013; Eveleigh et al., 2014; Eveleigh et al., 2014)

Raddick et al. (2013) found that "I am excited to contribute to original scientific research" was the largest primary motivation, selected by $40 \%$ of their respondents. We choose to highlight this 
study because they collected survey responses from almost 11,000 Galaxy Zoo volunteers - to our knowledge this is the largest sample size for a study exploring motivations to date.

Another important finding is that motivations change over time. Rotman et al. (2012) found that volunteers' initial interest in ecological citizen science projects stemmed from elements related to egoism, such as personal curiosity or previous engagement in science projects. Following their initial engagement, volunteers' ongoing participation was affected by factors such as recognition, attribution, feedback, community involvement and advocacy. Iacovides et al. (2013) found similar results in their study with Foldit and Eyewire volunteers. Interest in science and being pro-citizen science were identified as motives for joining a project. Sustained engagement was influenced by factors such as recognition, gaming elements, and team-play.

However a limitation of past research is that it has focused mostly on citizen science projects investigating the biological and physical sciences. Do these findings generalize to other kinds of citizen science projects?

\subsection{Citizen Psych-Science}

Psychology is an interesting research area to consider in relation to citizen science because ordinary citizens have always been involved in Psychology research, ever since Wilhelm Wundt founded the first psychology laboratory in 1879 to study human perception. However citizens' roles have typically been limited to that of research participants. The British Psychology Society provides the following definition for the term 'participant': "It is now common place to refer to a person who serves as a data source for research as a 'participant'. This recognizes their active role and replaces the term 'subject' which has been viewed as portraying people as passive recipients rather than active agents. While the extent of active 'participation' in the research over and above providing information will of course vary from one project to another, the use of the term 'participant' also serves to acknowledge the autonomy and agency of the individual in contributing to the research, and their right to withdraw at any time without penalty." (p.5, Code of Human Research Ethics)

Recently several psychology projects have emerged online that promote themselves as citizen science projects. Examples of psychology projects include:

- Lab in the Wild ${ }^{1}$ - volunteers take part in experiments that test their abilities and preferences when interacting with computers.

- Ignore That $^{2}$ - volunteers test how well they can ignore irrelevant information, to help researchers learn more about language structure and thought.

\footnotetext{
${ }^{1} \mathrm{http}: / / \mathrm{www}$. labinthewild.org

${ }^{2} \mathrm{http}: / /$ www.gameswithwords.org/IgnoreThat/
} 
- Mind Paths ${ }^{3}$ - volunteers play a game to define the semantic relationship between words, to help researchers improve semantic analysis.

- Mappiness $^{4}$ - volunteers chart their happiness alongside their location, to help researchers understand how people's happiness is affected by their local environment.

- $\quad$ Errordiary $^{5}$ - volunteers tweet about their everyday errors, to help researchers understand the range of errors that people experience in day-to-day life.

The website Scistarter $^{6}$ serves as a database of online citizen science projects and even has a section dedicated to Psychology projects.

\subsubsection{Personal data}

When considering citizen psych-science projects within the spectrum of citizen science projects, a crucial difference is the nature of the data. For example, in Galaxy Zoo, volunteers classify images of galaxies to help researchers understand how galaxies are formed. In Foldit, volunteers play a protein-folding game to help researchers understand how proteins are structured. In both of these examples, volunteers are contributing their analytical skills to analyze environmental data and to complete a research task, in the same way that the researcher would complete the same research task if they had the time and the resources to do so.

Citizen psych-science is different because the volunteer is the data. Psychologists are interested in understanding how volunteers (i.e. the participants) perform a certain task and/or their experiences of a certain event. On the one hand, it is possible that citizen psych-science also has the potential to engage volunteers in a much deeper way. Whereas with citizen science we learn about the world in which we live (third person narrative), with citizen psych-science there is a stronger likelihood of learning about ourselves, how we think and how we fit into the wider social world (first person narrative).

On the other hand, it is possible that some members of the public might feel apprehensive about taking part in citizen-psych science. They might feel uneasy with the idea of being assessed and possibly performing badly compared to others. We speculate that volunteers might have similar ambivalences about contributing to citizen science health projects (e.g. UK Flu survey ${ }^{7}$ ), again due to the personal nature of the data. Here a citizen-psych science project could be considered its own social setting, where people are concerned about the identity that they project to others. This

\footnotetext{
${ }^{3} \mathrm{https}: / /$ pybossa.socientize.eu/pybossa/app/Semantics/

${ }^{4}$ www.mappiness.org.uk

${ }^{5}$ www.errordiary.org

${ }^{6}$ www.scistarter.com

${ }^{7}$ https://flusurvey.org.uk/
} 
concern includes anticipated and unforeseen consequences in other settings too, e.g. online and offline, and professional and non-professional settings.

\subsubsection{One-time participation versus experiential studies}

In our literature review we identified a sense of community as an important factor for sustaining volunteers' engagement in virtual citizen science projects. Previous research suggests that feeling part of a project community can motivate volunteers to think creatively to solve project problems (Jennett et al., 2013). Feeling part of a project community can also motivate volunteers to gain new knowledge and skills as they take on more active project roles, such as moderators, editors, translators and community managers (Kloetzer et al., 2013).

Many psychology studies require one-time participation. This is particularly applicable to experimental studies where psychologists are attempting to measure participants' reaction times to a visual/auditory stimulus (e.g. Ignore That!) One-time participation is important because psychologists must try to avoid bias in their results. However it also means that it is unlikely that volunteers will feel a sense of project community, as the identities of other participants remain largely unknown.

An exception, however, are citizen psych-science projects where psychologists are interested in understanding people's experiences of a certain event. For example, in Mappiness, volunteers can contribute data whenever they experience feeling happy and results are collated on an online map. Similarly in Errordiary, volunteers can contribute data whenever they experience an error and results are collated on the Errordiary website. We suggest that in citizen psych-science projects such as these, where volunteers contribute more than once and contributions are publicly shared, there is potential for a sense of project community to develop amongst volunteers. Participants also have more of an opportunity to provide the researcher with feedback and to suggest new research directions.

\section{CASE STUDY: ERRORDIARY}

In our comparison of citizen psych-science projects with other types of citizen science projects, we identified the personal nature of the data as a key difference. To explore this issue further, we decided to investigate Errordiary in more depth. We chose Errordiary because in addition to fitting our citizen psych-science project criteria, it also has potential to offer a deeper level of participation from volunteers, e.g. communicating with the research team, feeling part of the project community. 


\subsection{History}

People make funny, frustrating and fatal errors on a daily basis. People can also create and apply strategies to avoid and mitigate error - this is called cognitive resilience. Psychologists at University College London Interaction Centre (UCLIC) started the Errordiary project in 2009 as a way of raising awareness of human error research. Over the years the project has gone through a number of different stages of development:

- 2009. Single tweeter. Dominic Furniss (@FaintSignals) started tweeting his own everyday errors whilst he was working for 6 months in Norway. The project he was working on investigated how the concept of resilience could apply to nuclear power plants, and part of his interest involved understanding error.

- 2010. Multi-tweeters but disconnected. Upon Dominic's return to the UK a small group of colleagues also interested in human error research thought this was a fun idea and decided to tweet their errors too.

- 2010. Multi-tweeters and connected. With a few of them tweeting errors from the department the researchers thought it was a shame that they were not linked. This is when they decided upon the hashtag \#errordiary to link the tweets. Searching for this hashtag would link all the tweets together. However, this was when they also realized that Twitter's search facility only showed tweets within the last two weeks, i.e. their ability to search was limited.

- 2011/12. The birth of the Errordiary website (see Figure 1). The researchers secured a small public engagement budget to develop a website which would harvest and store the \#errordiary tweets. They also wanted it to do the same for resilience strategy tweets which used \#rsdiary. In a second round of development they reduced repeat posts caused by retweeting and they created the ability to post directly to the website. The website started to attract attention from people outside of their department.

- 2013/14. Errordiary in healthcare. The researchers secured further public engagement money to run a three month competition to encourage healthcare professionals, people with diabetes and the general public to post errors and resilience strategies to the website. They also developed the Discovery Zone so that Errordiary became a hub of information and resources rather than just a stream of error posts and resilience strategy posts. This was the first direct attempt to reach out to communities that were not inherently interested in human error and resilience research. Due to this expansion they also had to tackle technical and ethical issues to do with informing tweeters and members of the website about how their information is being used. 


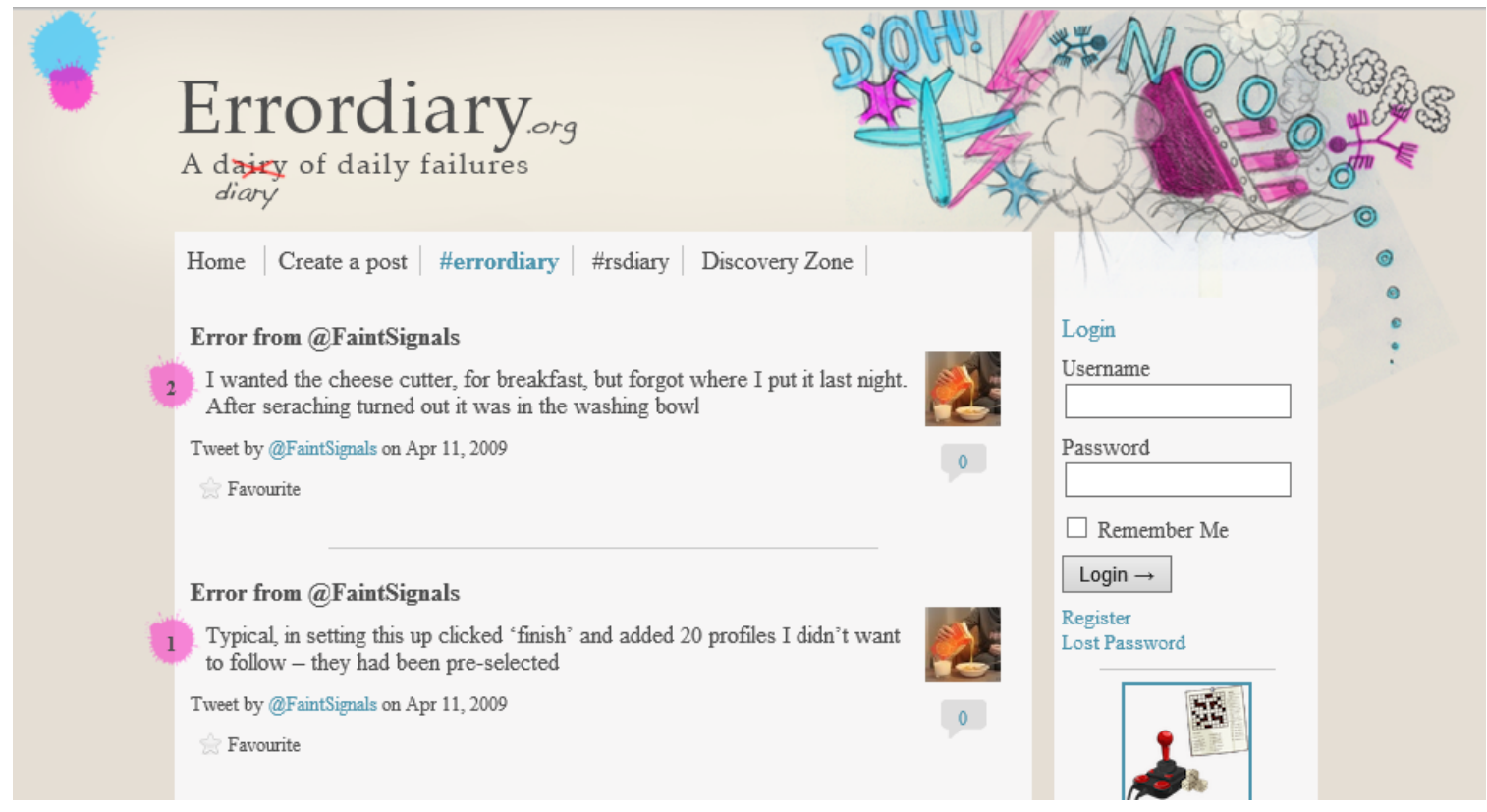

Figure 1. Screenshot of the Errordiary website.

Over 130 volunteers have contributed to the Errordiary project so far. The Errordiary website currently displays 2639 \# errordiary tweets and 417 \#rsdiary tweets, and these numbers continue to grow on a weekly basis.

\subsection{Teaching}

The Errordiary project originally started as a public engagement project. However, as the corpus of data has grown, the Errordiary researchers have seen new potential for its use. The project has allowed researchers to gain a better insight into the resilience strategies that people use (Furniss et al., 2012). It has also been used as a real-life data set when teaching students methods of categorizing error (Wiseman et al., 2012).

Wiseman et al. (2012) explain that error categorization techniques are limited because they were often created by a sole individual in order to categorise their own errors (e.g. Norman, 1981). This means that they do not always cover all types of error. This important lesson is highlighted when trying to apply categorization techniques to the errors in Errordiary, because the collection of errors have been contributed by a number of different users and represents a diverse selection of events that people consider to be errors.

The teaching exercise that uses Errordiary has gone through multiple iterations. In its first form, students were given work sheets after being introduced to the error classification scheme and 
asked to find instances of error on the Errordiary website that fit into each category. In later forms, this task has evolved to an interactive quiz played by the whole class on the board at the front of the room.

Teachers have found that students are able to easily classify many of the errors they find on Errordiary, but do encounter some that either fit into multiple categories, or none at all. These 'misfit errors' encourage debate amongst the students and with lecturers, which solidifies the students' understanding of the classification types. It also highlights to students the difficulties in dealing with real world data, which can at some times be messy, or lacking in information, and doesn't necessarily conform to your expectations at the outset.

\subsection{Massive Open Online Diary (MOOD)}

A further outcome of the Errordiary project has been the development of a new research method, known as a Massive Open Online Diary (MOOD). This is a hybrid method for data collection that combines traditional diary techniques with the benefits of an open online collaborative space (Gould et al., 2014). A MOOD invites individual contributors to make short diary-like posts on a particular topic of interest to both researchers and contributors. Contributions can be made through microblogging services, like Twitter, or through website-based interactions. These short, timely updates are then added to a publicly accessible corpus from which they can be searched, shared or analysed.

A MOOD has an advantage over traditional diary studies, because participants are able to gain an understanding of the research topic by reading through the online corpus, whereas in traditional diary studies participants are only aware of their own data. At the same time however, it is precisely the public availability of the online corpus that places psychologists in an ethical dilemma. For example, it is possible that users might tweet with the \#errordiary hashtag, unaware that their tweets were being gathered and then displayed on a public website. To tackle this issue, Gould et al. (2014) developed a procedure that balanced users' right to give consent against the technical constraints of building a protocol on a platform that was not designed with research ethics in mind. Whenever the harvester picked up a tweet with the \#errordiary hashtag from a new contributor, a tweet mentioning the user and a link to the privacy policy was sent. After reading the privacy policy, users had the option to opt-out, preventing Errordiary from capturing their tweets. They could do this either by confirming this through their Twitter account or by emailing the Errordiary researchers. Users who created an account at Errordiary.org were also given a privacy and data use policy to read.

The history of Errordiary's development above explains how it has evolved from a single user tweeting their errors into a MOOD. Along this journey, as Errordiary expanded, we needed to reflect on the best model of interaction that would suit our research aims, reflected the project's research ethic, and would encourage citizen psych-science participation by having the right 
balance between community, confidentiality and anonymity. Three broad models of interaction we considered included:

- A private model, which is epitomized by having all contributions completely hidden to anyone else other than the research team.

- An anonymous model, which is epitomized by having contributions on display to other participants but by having no names or identifiers in the contributions.

- An open model, which is epitomized by having all contributions on display and linked to a user name or Twitter handle.

From a naïve point of view one might assume that private and anonymous models might be most appropriate for citizen psych-science projects, because personal data is protected. However, the private model was considered unsuitable for Errordiary because it did not provide the potential for community that we wished to engender. Also, people could not learn from reading each other's posts. The anonymous model also eroded the sense of community but it also provided its own challenges: what level of anonymity is promised and how is this secured? For example, removing names from posts from Twitter is inadequate as simple online searches could reveal the original post and its author. It is far riskier to promise anonymity that can be broken, than it is to go with an open model from the start. With the private and anonymous models we also thought that participants might feel disenfranchised if they were not properly recognized for their contributions. The private and anonymous models also conflicted with the underlying ethic of our project, i.e. we were trying to encourage people to talk more openly and honestly about error hiding names and talking about errors in secret conflicted with this. The open model recognized contributors by name but within this there was concern that people might feel inhibited by being linked to their personal contributions. To counteract this we advised people, on the Errordiary website, that they may wish to setup an account with a pseudo name to distance themselves from their other professional and online identities.

\section{STUDY 1: MOTIVATIONS OF ERRORDIARY VOLUNTEERS}

The first study we conducted was an interview study with Errordiary contributors. Our aim was to gain an impression of Errordiary use and why people currently participate in the project. In particular, we were interested in understanding factors that encouraged and/or discouraged participants from contributing to the Errordiary project.

\subsection{Method}

Eight participants ( 5 female, 3 male) were recruited via an advert on the Errordiary website and twitter feed. They were interviewed during August 2013. Interviews were conducted over Skype or in person. Participants were asked several questions about their experience of Errordiary- e.g. what motivates you to take part? What do you feel you learn from it? Each interview was semistructured and took approximately 30-45 minutes. All interviews were audio recorded. At the end 
of the interview, participants were debriefed and received a $£ 5$ gift voucher for taking part. Interview transcripts were analysed using 'thematic analysis' - a qualitative method for identifying, analysing and reporting patterns such as themes within a data set (Braun and Clarke, 2006).

\subsection{Results}

\subsubsection{Backgrounds and Motivations}

All 8 participants were researchers: 6 participants carried out research related to errors and 2 participants carried out research not related to errors. All 8 participants also had a personal connection to UCLIC: they had either studied/worked at UCLIC themselves (4 participants), they had collaborated on a project with UCLIC researchers (2 participants), or they were close friends with UCLIC researchers (2 participants).

Participants contributed to Errordiary for many reasons. The project was described as a "nice idea", "interesting" and "intriguing" (3 participants). Some participants expressed a general desire to contribute to research ( 2 participants); while others specified that they were interested because the project was related to their own research ( 2 participants). In addition, contributing required very little effort: "I was already sharing errors on Twitter, now it's just adding a hashtag" (2 participants).

Social pressures also played a crucial role in people's motivations. Some participants joined because they wanted to be supportive of their colleagues at within the department ( 2 participants), or they knew people that were involved in Errordiary and they wanted to be part of "the club" (2 participants). Furthermore, sharing errors was described as "fun" and "humorous" (3 participants). Participants described how they tried to compose tweets that were "funny" because contributing to the project wasn't just about sharing data, it was about sharing amusing stories. As one participant explains "I think $50 \%$ of people that take part are error researchers, they have a professional interest. The other $50 \%$ probably like sharing stories, it's quirky, self-deprecating. There is a humorous, entertainment aspect to the project. Reading and writing tweets is a form of entertainment." Similarly, another participant says "I generally post stuff that I find funny and that I think other people will find funny."

A couple of participants described how they had set up a separate Twitter account just for the purpose of contributing to Errordiary. One participant did this because she wanted to avoid "spamming" or "boring" her followers with error tweets that were not funny. Another participant did this because she didn't want to tweet about errors from her work Twitter account. She explains that she wanted to "maintain some level of privacy... even though contributing to Errordiary is a professional activity, often my tweets are about my personal life." 
Six participants described how they had recruited other users by telling them about the project, or nudging them to add the hashtag if they tweeted an error. Two participants described how they had tried to recruit others but had been unsuccessful. They thought that perhaps their colleagues did not contribute because they did not use Twitter often and/or errors were not the focus of their research.

\subsubsection{Frequency of Contributions}

\subsubsection{1 \#errordiary}

When asked about how often they contributed an error to Errordiary (\#errordiary), answers varied from once a week (1 participant), once a month (2 participants) and once every 6 months (1 participant). Many participants described their contributions as "occasional", saying that they felt unable to put frequency to their contributions (4 participants). As one participant explains "I go through a period of not contributing for weeks and then remembering it exists."

Some participants said that they forgot to contribute because they were not regular Twitter users. As one participant explains "I'm not a frequent user of Twitter so sometimes I forget, as I'm not in the habit of posting there". Similarly, another participant says "Sometimes I forget to put it on Errordiary, I don't use twitter as regularly as others."

Another issue was the event-driven nature of contributing. A person must experience an error, but this might not happy very often: "I don't go looking for things, it's just when I come across something, so it's very much event driven" (1 participant). As well as experiencing an error, it is necessary for people to consciously recognize that they have made an error, which does not always happen: "I make lots of errors, but might not recognise them all the time" (1 participant).

Participants' desires to be funny and interesting also impacted their contributions. As one participant explains "Sometimes when I forget to do it straight away, later on it doesn't feel that funny anymore and I think 'oh well I missed the chance". Similarly, another participant says "I get tired of posting mundane things".

A further issue was the limited number of characters that a person can write in a tweet: "Some errors I've made, I would have liked to tweet but it's hard to put in 140 letters. This is one of the weaknesses, you can't tell more complicated stories" (1 participant).

\subsubsection{2 \#rsdiary}

Resilience diary contributions (\#rsdiary) were less frequent compared to Errordiary contributions (\#errordiary). Five participants had never contributed to resilience diary. Two participants occasionally posted if they thought that they had developed a strategy that someone else might find useful. One participant contributed every few weeks because resilience was their research focus. 
There were many reasons why \#rsdiary contributions were less popular. Participants explained that they didn't think about resilience strategies very often. Whereas errors were noticeable and often stood out, resilience strategies were often things that they had been doing for quite some time (3 participants). Some participants felt that their resilience strategies were too long or complicated to explain in a tweet (4 participants). Also resilience strategies were viewed as mundane and not worth sharing, whereas errors were more fun (2 participants).

A further issue was that the \#rsdiary hashtag was not as self-explanatory as the \#errordiary hashtag. One participant described how she was reluctant to use it because "If I use the \#rsdiary hashtag my friends won't know what it means". Another participant said that she didn't tweet about her strategies because "I don't remember the hashtag".

\section{STUDY 2: EXPLORING POTENTIAL TO ATTRACT NEW COMMUNITIES}

Study 1 allowed us to gain some valuable insights into the motivations and experiences of Errordiary volunteers. In line with past research, we found that our participants were motivated by their desire to contribute to original research, because they wanted to help others and feel part of a team and because they enjoyed the research task. In fact all 8 of our participants were researchers themselves, with 6 out of the 8 carrying out error-related research. But what about people that do not have an intrinsic interest in error research, how can Errordiary engage these people in their project?

For our next study we decided to explore the potential of attracting new communities to the project. We decided to focus on 3 groups: (1) medical professionals, (2) people with diabetes and (3) the general public. Again we were interested in understanding factors that might encourage and/or discourage participation in the Errordiary project. We also wanted to explore what these groups thought about everyday errors and medical errors. For example, if we accept that 'to err is human' then should we be more accepting of medical staff who err? More specifically for the medical professionals: do they feel there is a blame culture within healthcare and can they become more mindful of error by sharing trivial examples of it? For people with diabetes we wanted to learn about the errors and issues they face in diabetes management so solutions could be considered. For members of the public we wanted to contrast these medical errors with everyday domestic errors and open the door to serendipity, i.e. we did not know which members of 'the public' would get involved. For all three groups we wanted to encourage debate about whether more serious and everyday errors were actually that different.

On the flipside to human error we also wanted to know whether these groups engaged with the idea of resilience strategies and whether they thought this new concept we were introducing could be useful. For medical professionals we had already completed an observational study to reveal resilience strategies amongst nurses (Furniss et al., 2011); using a crowdsourcing technique had the potential to collect many more examples at a much lower cost. For people with diabetes we 
wanted to learn the informal ways they manage their diabetes and reduce the likelihood of error. For both of these communities we wanted to distil and share best practices, so the broader community could learn about the best resilience strategies that had been shared. Again the public group was included to provide interesting everyday examples to enhance the broader data set, and again to open the door to serendipity.

\subsection{Method}

We conducted three focus groups during September 2013 with three groups:

1. Medical professionals - focus group with approximately 20 participants

2. People with diabetes - focus group with 6 participants

3. General public - focus group with 7 participants

During each session the facilitator introduced participants to the topics of error and resilience strategies. Participants were then asked to discuss the potential benefits and limitations of sharing errors and resilience strategies on an online website such as Errordiary.

The medical professionals' focus group was conducted in a hospital during the staff's lunch hour. Approximately 20 people attended and food and drink were provided for all attendees.

Two smaller focus groups were conducted on the university campus. We recruited participants via adverts posted on the university's psychology subject pool and several different medical forums and social media sites. Six people attended the 'people with diabetes' focus group and 7 people attended the 'general public' focus group. Food and drink were provided and participants were paid $£ 20$ for their participation.

Written notes were made during the focus groups and these notes were analysed using thematic analysis.

\subsection{Results}

\subsubsection{Medical Professionals}

The medical professionals group thought that the idea of Errordiary was interesting but they would be reluctant to contribute themselves. Several participants were worried that sharing errors in an open forum, particularly work errors, could have legal implications. They would need to check with their legal department to see if they were allowed to contribute.

Some participants also said that they would not want to share errors on an open forum - reading about the errors that doctors and nurses make could worry patients. One participant used the analogy that if you were getting on a plane you would not want to know if your pilot makes mistakes as you would not want them flying the plane. 
Time was another concern. Participants commented that they worked long hours and were unsure whether they would have time to participate in a website such as this.

Overall the general consensus was that if they were to share errors it would need to be for a specific audience, with a specific reason for value, and it would need appropriate screening, anonymous and closed. Also the language would need to be framed in a more positive way rather than "error" or "failure".

\subsubsection{People with Diabetes}

Participants in the diabetes group liked the idea of Errordiary. One participant commented "It would be nice to have somewhere to post those stupid things you've done." Another participant talked about how Diabetes UK has a Facebook page where lots of people share messages about their management of their blood sugar, so it does appear that people enjoy talking about diabetesrelated errors and already do share such errors online.

Categorization was described as "massively important." If they visited the Errrodiary website and wanted to view just diabetes-related errors and strategies, they wanted an easy way to be able to do this, e.g. a search function or a separate 'diabetes' page. Another comment was that although reading people's errors might be amusing, they wanted to read solutions to the errors too. It was suggested that a good idea would be to directly link resilience strategies (or other kinds of solutions) to particular types of errors.

A further issue related to attitudes towards social media. One participant said that even though she thought it sounded like a good idea she would not take part herself as she doesn't like to use much social media unless it has a very clear purpose. Another participant commented that he would not take part because he is not a Twitter user.

\subsubsection{General Public}

The general public group discussed how they could see two purposes for sharing errors online:

(1) to note your own mistakes so others can learn, and (2) as a kind of anonymous confessional, to let off steam about mundane things.

"Error" was viewed as too broad a category and participants thought that it would need to be organized into different types, e.g. home, office, relationships, leisure. Some participants discussed how they would want to see "real problems and real solutions." For example, resilience strategies could be suggested as responses to errors. Other participants discussed Errordiary in terms of its potential for entertainment value. Suggestions included that it would be good if you could see errors posted by celebrities, if you could rank the severity of an error, vote for "worst error of the day", maybe click an "I did that too" button. 
Several participants raised concerns about how the site would be monitored. The general consensus was that researchers would need to control the types of errors being posted, removing any "nasty replies" and spam. One participant talked about the need to "control for trolls". Another participant talked about how there could be a breach of confidentiality, as some users might want to "name and shame."

\section{GENERAL DISCUSSION}

Since its early beginnings in 2009 where there was just a single tweeter, the Errordiary project has transformed and developed, attracting contributions from over 130 volunteers. In our research we wanted to understand factors that might encourage and/or discourage participation in the Errordiary project. We conducted interviews with eight Errordiary contributors to explore their motivations (Study 1) and we conducted three focus groups to explore the potential of attracting new communities to the project (Study 2). Our findings highlight that one of the main ways that Errordiary, a citizen-psych science project, differs from citizen science projects in other domains (e.g. the physical and biological sciences) is the personal nature of the data.

Previous citizen science research suggests that the presence of a project community can help to motivate contributions (Iacovides et al., 2013; Reed et al., 2013), encourage creative outputs (Jennett et al., 2013) and lead to greater learning (Kloetzer et al, 2013). In the case of Errordiary, where personal data is involved, we observed that participants found it "fun" to share their errors with others. Social factors such as "helping" and "sharing" encouraged them to join and contribute to the project. At the same time however, we found that social factors could also inhibit participation. Our Study 1 results revealed that Errordiary participants were sometimes put off posting because they thought others might view their contribution as "mundane" or "not funny." This is in line with past research investigating privacy perceptions of computer-mediated communication: users are more concerned with how others will perceive their online communications if they view the information being communicated as "personally defining" (Adams \& Sasse, 2001). The first person narrative associated with citizen psych-science projects means that contributions are potentially much more personally defining. Contributors to Errordiary can also contribute errors reported in the media and which they observe in others, this introduces a third person narrative that is less personally defining, and arguably more comfortable for people to share.

A further issue is that Errordiary uses a hybrid method for data collection. In most citizen science projects people contribute via a dedicated website or platform, within the "safety" of being among like-minded others who share their interests. Errordiary differs because it utilizes Twitter for data collection. This means that when a participant uses the \#errordiary or \#rsdiary hashtags, their contribution is visible to all of their Twitter followers, who may or may not know about the project. This heightens the perceived risk of negative perceptions from others. A couple of participants even described how they had set up a separate Twitter account just for the purpose of 
contributing to Errordiary, thereby making a trade-off between the convenience of maintaining just one account and protecting their online identity.

Our second study revealed that fears about confidentiality and negative replies from others were also reasons why people might be reluctant to volunteer in the first place. Because errors are a sensitive topic, particularly in certain professions (e.g. medical), some people were worried that they could get in trouble at their work or that the site could attract "trolls". These results reveal that the risks associated with sharing errors (e.g. negative perceptions from others, being viewed as incompetent) may counteract a person's general goodwill to help researchers. To alleviate privacy concerns, it will be important to inform users how their personal data will be used and the measures in place to make sure their data remains confidential, e.g. anonymity, secure data storage (Adams \& Sasse, 2001). It may be that for this user group, where the associated risks of sharing errors is much higher, a private model might be more suitable than an open model.

Another key finding from our research was that Errodiary is centered on quite a niche topic. Current contributors to Errordiary are mostly those that are already interested in error and/or have personal ties to researchers involved in the project. To attract new users to Errordiary, our focus group results suggest that the project would need to find ways to make errors seem more interesting (e.g. voting features) or more useful (e.g. ability to search for information).

Further barriers to participation include accessibility, time, and attitudes to social media. Some participants described forgetting to contribute. One reason for this is that contributions are eventdriven. People cannot contribute whenever they wish - it has to be after they have committed an error or used a resilience strategy. Those that were regular twitter users were more likely to remember to contribute. As one participant describes, "I was already sharing errors on Twitter, now it's just adding a hashtag." Possibly there are ways to remind infrequent contributors ("dabblers") about the project; for example, emailing updates via a project mailing list and publicizing scientific outcomes (Eveleigh et al., 2014).

In relation to factors that may obstacle initial participation in Errordiary, some were concerned about the time they would have to invest in the project. Not liking Twitter and not liking social media sites were also suggested as reasons why some people would be reluctant to volunteer. Again it appears that the nature of the contribution (sharing on Twitter) may counteract a person's general goodwill to help researchers. If people decide to give up their free time to contribute they want the project to have some kind value to them (e.g. entertainment or educational) and it should not come at a personal cost (e.g. negative perceptions from others).

Overall these findings reveal several interesting insights concerning the spectrum of citizen science, and pros and cons in using Twitter for data collection. The Errordiary project is currently changing from being an online archive of error to a portal for engaging with and learning about error. This includes a 'Discovery Zone', allowing volunteers to explore research, media and games related to errors. It is now also possible for volunteers to login and contribute via the 
website, so the project is no longer restricted to Twitter users only. We plan to explore how these changes impact volunteers' experiences in future research.

\section{CONCLUSION}

When considering citizen psych-science projects within the spectrum of citizen science projects, a crucial difference is the origin of the data. Psychologists are interested in understanding how volunteers (i.e. the participants) perform a certain task and/or their experiences of a certain event. Consequently, the data is immediately more personal to the volunteer because they themselves are the topic being studied. In this paper we conducted two research studies to explore motivations and barriers for contributing to Errordiary, a citizen psych-science project where participants tweet about their everyday experiences of human error.

Our findings suggest that the personal nature of contributions in citizen psych-science projects can influence volunteers' participation in positive and negative ways. In particular, the perceived risks associated with sharing errors on Errordiary may counteract a person's general goodwill to help researchers. It is important that scientists help citizen psych-science volunteers to feel like they are in a safe space to contribute their personal experiences towards research, without fear of negative perceptions from the psychologists, the project community or the public in general. We suggest that citizen psych-science projects can do this by considering the most suitable model of interaction (e.g. private, anonymous or open), clearly communicating the purpose of their research, and allowing flexibility so participants can anonymise their contributions to protect their identity. In future research it would be interesting to explore the extent to which our findings with Errordiary generalize to other citizen psych-science projects, as well as other kinds of citizen science projects that involve contributions of personal nature (e.g. health).

\section{ACKNOWLEDGEMENTS}

Cox, Furniss, Gould, Iacovides and Wiseman are funded by the CHI+MED project, EPSRC grant EP/G059063/1. Cox and Jennett are funded by the Citizen Cyberlab project, EU FP7 \#317705.

\section{REFERENCES}

Adams, A. and Sasse, M. A. (2001). Privacy in multimedia communications: Protecting users, not just data. In A. Blandford, J. Vanderdonkt \& P. Gray (Eds.), People and Computers XV - Interaction without frontiers. Joint Proceedings of HCI2001 and ICM2001, pp. 49-64. Springer.

Braun, V. and Clarke, V. (2006). Using thematic analysis in psychology. Qualitative research in psychology 3 (2), $77-101$.

Causer, T. and Wallace, V. (2012). Building a volunteer community: Results and findings from Transcribe Bentham. Digital Humanities Quarterly 6 (2). 
Code of Human Research Ethics. (2010). The British Psychological Society [On-line] http://www.bps.org.uk/publications/policy-and-guidelines/research-guidelines-policy-documents/researchguidelines-poli

Crowston, K. and Prestopnik, N.R. (2013). Motivation and data quality in a citizen science game: A design science evaluation. Proceedings of HICSS 2013, 450-459.

Eveleigh, A., Jennett, C., Blandford, A., Brohan, P. and Cox, A. L. (2014). Designing for dabblers and deterring dropouts in citizen science. Proceedings of CHI '14, ACM Press, 2986-2994.

Furniss, D., Back, J. and Blandford, A. (2012). Cognitive resilience: Can we use Twitter to make strategies more tangible? Proceedings of ECCE 2012, 96-99.

Furniss, D., Blandford, A., Mayer, A. (2011). Unremarkable errors: Low-level disturbances in infusion pump use. Proceedings of BCS-HCI 2011, pp.197-204. Swindon, UK: BCS.

Gould, S. J. J., Furniss, D. J., Jennett, C., Wiseman, S., Iacovides, I. and Cox, A. L. (2014). MOODs: building massive open online diaries for researchers, teachers and contributors. Proceedings of CHI EA '14, ACM Press, 2281-2286.

Iacovides, I., Jennett, C. Cornish-Trestrail, C. and Cox, A. L. (2013). Do games attract or sustain engagement in citizen science?: A study of volunteers motivations. Proceedings of CHI EA '13, ACM Press, 1101-1106.

Jennett, C., Eveleigh, A., Mathieu, K., Ajani, Z. and Cox, A. L. (2013). Creativity in citizen cyberscience: All for one and one for all. ACM WebSci 2013, 'Creativity and Attention in the Age of the Web' workshop.

Kloetzer, L., Schneider, D., Jennett, C., Iacovides, I., Eveleigh, A., Cox, A.L. and Gold, M. (2013). Learning by volunteer computing, thinking and gaming: What and how are volunteers learning by participating in Virtual Citizen Science? Conference abstract at ESREA 2013.

Norman, D.A. (1981). Categorisation of action slips. Psychological Review 88 (1), 1-15.

Nov, O., Arazy, O. and Anderson, D. (2010). Crowdsourcing for science: Understanding and enhancing SciSourcing contribution. Proceedings of CSCW 2010, focus group on the 'Changing Dynamics of Scientific Collaborations'.

Nov, O., Arazy, O., and Anderson, D. (2011). Dusting for science: motivation and participation of digital citizen science volunteers. Proceedings of iConference 2011, 68-74.

Raddick, M.J., Bracey, G., Gay, P.L., et al. (2010). Galaxy Zoo: Exploring the Motivations of Citizen Science Volunteers. Astronomy Education Review 9 (1), 010103.

Raddick, M.J., Bracey, G., Gay, P.L., et al. (2013). Galaxy Zoo: Motivations of Citizen Scientists. arXiv preprint, arXiv:1303.6886.

Reed, J., Raddick, M.J., Lardner, A., and Carney, K. (2013). An exploratory factor analysis of motivations for participating in Zooniverse, a collection of virtual citizen science projects. Proceedings of HICSS $2013,610-619$.

Rotman, D., Preece, J., Hammock, J., Procita, K., Hansen, D., Parr, C., Lewis, D. and Jacobs, D. (2012). Dynamic changes in motivation in collaborative citizen-science projects. Proceedings of CSCW 2012, ACM Press, 217-226.

Wiggins, A., and Crowston, K. (2011). From conservation to crowdsourcing: a typology of citizen science. Proceedings of 44th Hawaii International Conference on System Sciences (HICSS '10).

Wiseman, S., Gould, S. J. J., Furniss, D. and Cox, A. L. (2012). Errordiary: Support for teaching human error. 'A contextualized curriculum for HCI' workshop at CHI 2012. 